\title{
Investigation of the Effects of Leg Dominance on Cross-Transfer of Flexibility after a Unilateral Treatment with Foam Roller - A Pilot Study
}

\author{
Katerina Daskalaki*, George Pafis, Asimenia Gioftsidou, Anastasia Beneka, Evangelos Bebetsos, \\ Paraskevi Malliou
}

Department of Physical Education and Sport Science, Democritus University of Thrace, Greece

Received March 3, 2020; Revised April 24, 2020; Accepted May 3, 2020

Copyright $(\mathcal{C} 2020$ by authors, all rights reserved. Authors agree that this article remains permanently open access under the terms of the Creative Commons Attribution License 4.0 International License

\begin{abstract}
Cross Transfer" (CT) refers to the enhancement of performance of a non-trained limb after unilateral training of its opposite homologous limb. Another concept that sometimes is investigated when examining CT is "asymmetrical transfer" (AT), term that implies that the magnitude of transfer is not even for the two directions (i.e. from dominant to non-dominant limb and vice versa). While the existence of AT for strength or skills is already investigated, according to our knowledge previous research has not examined if laterality has an influence on the magnitude of CT of flexibility (CToF) in the lower limbs. The aim of the present study was to examine if a unilateral application of Foam Roller (FR) to the ankle plantar flexors of female exercisers would cause a CToF to their untrained limb and if leg dominance has an influence on the magnitude of CToF (i.e. if the CToF is greater from the dominant to the non-dominant limb or vice versa). According to the statistical analysis, the FR protocol used was effective in increasing the participants' ipsilateral and contralateral ankle range of motion (ROM) independently of limb dominance, indicating that when referring to $\mathrm{CToF}$ through FR the transfer will probably occur symmetrically. This finding adds to the literature new data about CToF of the lower limbs in relation to laterality. If these results could be expanded by future researches and could be established that unilaterally injured persons benefit from CToF independently of limb dominance, that would be a major contribution to the field of rehabilitation.
\end{abstract}

Keywords Cross Education, Limb Dominance, Laterality, Self-myofascial Release, Asymmetrical Transfer, Flexibility, Weight Bearing Lunge Test

\section{Introduction}

"Cross Transfer" (CT) is called the existence of exercise-induced adaptations and the enhancement of performance of a non-trained limb (upper or lower) after unilateral training of its opposite homologous limb. Other terms used to describe this phenomenon are "cross education", "cross-over effect", "interlateral", "bilateral", "interlimb" or "lateral" transfer and these terms usually refer to CT of strength, skills or flexibility [1-4). Another concept that sometimes is investigated when examining CT is the so-called "asymmetrical transfer" (AT), term that implies that the magnitude of transfer is not even for the two directions (i.e. it differs from dominant to non-dominant and from non-dominant to dominant limb or from right to left limb and vice versa) [1]. When referring to CT of strength or skills of the upper limbs, in some researches it has been argued that adaptations to the non-trained limb are greater when the dominant limb (i.e. the most proficient) receives the training $[1,5,6]$ or that can be totally absent after left-arm training [5] revealing, in this way the existence of AT, while in others that CT of strength is bi-directional (symmetrical) between dominant and non-dominant sides after unilateral training of either the dominant or non-dominant limb in right-handed individuals [7]. Concerning CT of skills in the lower limbs, a study concluded that there was AT of a learned skill (moving a cursor towards targets using ankle movements) since CT occurred only from the non-dominant to the dominant side and not vice versa [8] while in a different study it was found that they didn't exist side-specific differences in the amount of CT from the right to the left side or vice versa in a novel ankle isometric force control skill [9]. As for studies that investigated leg dominance in relation to $\mathrm{CT}$ of locomotor 
skills, differences in the amount of CT from the right to the left side or vice versa weren't found neither in a newly acquired locomotor skill (stepping over an obstacle on a treadmill) [10] nor during walking with a new motor learning task for one limb [11].

The investigation of CT is a promising step in the field of rehabilitation, since it could provide an alternative solution for the partial maintenance of some parameters of the physical condition during the period of immobilization of an injured limb through the training of its opposite limb $[1,6,12]$. Concerning AT, it also consists an important parameter that should be investigated in order to verify if differences should be expected depending on which limb receives the training (the dominant or the non-dominant). In this way, by understanding what it should be expected after unilateral training of the uninjured dominant or non-dominant limb in relation to $\mathrm{CT}$, therapists would be able to estimate more accurately the time-course of the rehabilitation [13].

While the existence of AT for strength or skills after unilateral training is already investigated, according to our knowledge previous research has not examined if laterality has an influence on the magnitude of $\mathrm{CT}$ of flexibility $(\mathrm{CToF})$ in the lower limbs. Previous researches that examined CToF of the lower limbs acutely after a protocol with static or dynamic stretching [14] or foam rollers $[2,15]$ revealed a statistically significant increase of the contralateral flexibility, but they only trained the dominant limbs without examining the $\mathrm{CToF}$ in relation to AT. Considering that the recovery of ROM and flexibility is one of the first goals of a rehabilitation program after an athletic injury in the lower limbs [16], the maintenance of ROM of the injured limb through the training of the uninjured one would probably speed up the recovery of flexibility and it would be helpful to have information on what should be expected in relation to CToF by firstly examining healthy adults.

\section{Objectives}

The aim of the present study was to examine if an application of Foam Roller (FR) to the ankle plantar flexors of one limb (trained, ipsilateral limb) would cause a CToF to the other (untrained, contralateral) limb and if leg dominance has an influence on the magnitude of CToF (i.e. if the $\mathrm{CToF}$ is greater from the dominant to the non-dominant limb or vice versa). In order to gain more information on the topic, the time course of these effects were investigated up to $20 \mathrm{~min}$.

\section{Materials and Methods}

\subsection{Subjects}

38 women, gym members from 7 different gyms of
Athens participated in the research. Inclusion criteria were the healthy profile of the subject, normal BMI and age range between 30-50 years. Exclusion criteria were serious medical issues, musculoskeletal problems in the lower limbs and in the wrists or any other musculoskeletal disorder during the last 12 months that could affect negatively the health of the participants or their ability to properly follow the procedure. The participants were divided to an experimental ( $\mathrm{n}=19$, age: $41.07 \pm 5.58$ years, height: $165.58 \pm 6.48 \mathrm{~cm}$, mass: $58.74 \pm 4.25 \mathrm{~kg}$, BMI: $21.41 \pm 1.52 \mathrm{~kg} / \mathrm{m}^{2}$, physical activity per week: $4.05 \pm 2.21$ hours) and a control group ( $\mathrm{n}=19$, age: $41.18 \pm 5.46$ years, height: $169.21 \pm 5.07 \mathrm{~cm}$, mass: $59.03 \pm 3.68 \mathrm{~kg}$, BMI: $20.70 \pm 1.29 \mathrm{~kg} / \mathrm{m}^{2}$, physical activity per week: $4.08 \pm 1.68$ hours). All the participants of the experimental group would apply FR in their least flexible limb (according to baseline measurements), while the participants of the control group would remain seated in a comfortable position on a mat on the floor with their back against the wall and their legs totally relaxed. In order to produce results according to limb dominance, in the stage of the statistical analysis, the experimental group was further subdivided in two subgroups according to leg dominance, i.e. "the dominant-leg group" $(\mathrm{n}=8)$ whose least flexible limb -in which they applied FR- was the dominant $(n=8$, age: $40.37 \pm 5.07$ years, height: $165.75 \pm 9.02 \mathrm{~cm}$, mass: $59.44 \pm 4.43 \mathrm{~kg}$, BMI: $21.62 \pm 1.59 \mathrm{~kg} / \mathrm{m}^{2}$, physical activity per week: $4.56 \pm 2.29$ hours) and the "non-dominant leg group" whose least flexible limb was the non-dominant $(\mathrm{n}=11$, age: $41.59 \pm 6.11$ years, height: $165.45 \pm 4.32 \mathrm{~cm}$, weight: $58.23 \pm 4.25 \mathrm{~kg}$, BMI: $21.26 \pm 1.54 \mathrm{~kg} / \mathrm{m}^{2}$, physical activity per week: $3.68 \pm 2.18$ hours). All participants, apart from one participant of the "non-dominant leg group", indicated as dominant leg their right leg. This is similar to Yen et al. [9] that the left-footed subject wasn't excluded from the analysis since the research was focused on dominant/non-dominant leg rather than right/left leg.

The Research Ethics Committee of Democritus University of Thrace approved this study and informed consent was obtained from the study participants.

\subsection{Definition of Limb Dominance}

The dominant limb was defined by the answer on the question: "If you would shoot a ball on a target, which leg would you use to shoot the ball?" that has been shown to be the most accurate question in order to define leg dominance in bilateral mobilizing tasks [17].

\subsection{Weight-bearing Lunge Test}

Upon arrival the subjects were guided on how to correctly perform a weight-bearing lunge test against the wall (Figure 1). Since in the present research the way of executing this test was a combination of instructions found in previous articles [2,18-20] but also included some additional instructions and slight modifications not present in previous researches, the whole procedure will 
be described in detail: the participants were instructed to place their right foot on the measure attached to the floor as far away from the wall possibly and to try to touch the wall with their knee flexed without lifting their heel from the ground. They were instructed on how to adjust the distance from the wall, sliding the foot a little bit forward or backwards, till they reached their maximum capacity of keeping the posture indicated. Additionally, their trunk should be erect, the pelvis should be no rotated, their knee had to travel to a straight line till the wall, they were permitted to touch the wall with their hands and the other leg was placed, slightly bent or stretched, in a comfortable distance behind. They were given time to find their best shot using all the attempts they needed and when they were ready, they would inform the researcher to proceed with the measurement whose score was the distance from the big toe to the wall. To ensure that the heel was firmly on the ground, the researcher placed a resistance band under the heel of the subject and stretched it -method proposed by Halperin et al. [19]. If the elastic band snapped away, the attempt wouldn't be successful and the participant would have to try again. After the first successful attempt, the participant was asked to remain in the attained posture, the score was written down, and she was asked to try to move the foot a little bit backwards, so as to check if what she claimed to be her best, actually was. In case it was proven that the participant could manage even more, the researcher would give her additional feedback till the achievement of the highest possible score. The same procedure was followed for the left leg.

These first scores were the baseline measures (before the application of the FR protocol) and all the actions described during this first measurement also assured that the procedure had been properly learned. In all subsequent measurements (post-intervention) the first foot to be measured would be always the least flexible in which the FR was applied.

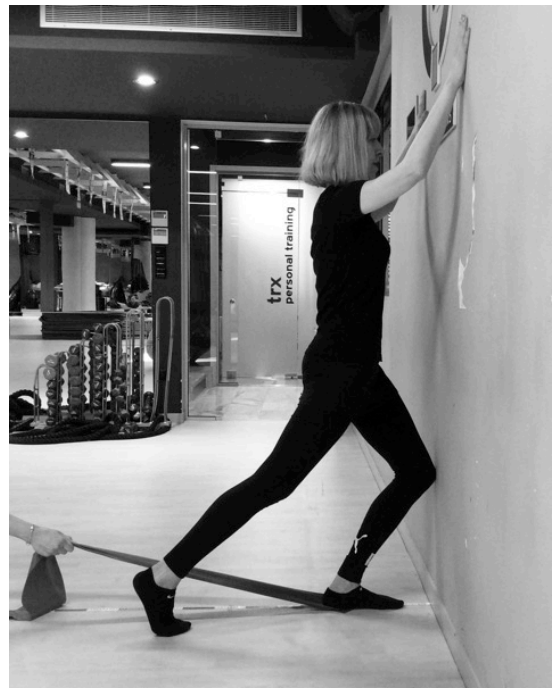

Figure 1. Weight-bearing Lunge Test

\subsection{Foam Rolling applied Protocol}

The FR used was a smooth FR made by foam (AMILA 48088) 15X31. The protocol was first demonstrated and verbally described in detail to the female exercisers of the experimental group by the researcher. It had a total duration of $5 \mathrm{~min}$ and $40 \mathrm{~s}$ and was distributed in three uninterrupted phases as following:

A) 3 bouts of $40 \mathrm{~s}$ of continuous rolling with $20 \mathrm{~s}$ break between bouts.

B) 3 bouts of $20 \mathrm{~s}$ of focused pressure in a "hot spot" with $20 \mathrm{~s}$ break between bouts.

C) 1 bout of $40 \mathrm{~s}$ of continuous rolling engaging the whole calf musculature.

The participants were guided to sit on the floor with their palms beside their hips, to place the foot indicated to them on the FR in the height of achilles tendon, to keep the other leg crossed above it, to sustain their weight with their palms and to lift their pelvis from the floor (Figure 2).

For phase $\mathrm{A}$ and $\mathrm{C}$ the participants would have to roll the FR from the height of achilles tendon till just below the height of the popliteal fossa and when they reached just before it to proceed rolling to the opposite direction. The participants realized for each bout totally 8 rolls, in a constant rhythm given by the researcher who was loudly counting from 1 to 5 , rhythm that corresponded to $5 \mathrm{~s}$ for the rolling in each direction. For phase A the subjects were guided to roll on the lateral aspect of the plantar flexors for the first bout, the middle for the second and the medial for the third as proposed by Kelly \& Beardsley[2]. For phase B they were guided to exert sustained pressure in a "hot spot" identified by them during phase A and also to involve some micro-movements back and forth around that specified spot, again following the logic of phase A, that is, working in 3 different aspects of the calf in each bout. Finally, in phase $\mathrm{C}$ they should roll again the entire calf musculature up and down, including also the "hot spots" that they had treated during phase B or if they failed to do that to proceed focusing on the medial aspect of the calf.

During the whole protocol they were advised to exert as much pressure necessary as to feel a tolerable amount of pain, to lessen the pressure exerted or modify the angle in a manner so as not to suffer if the pain became intolerable and to use their breath in a way to relax while rolling.

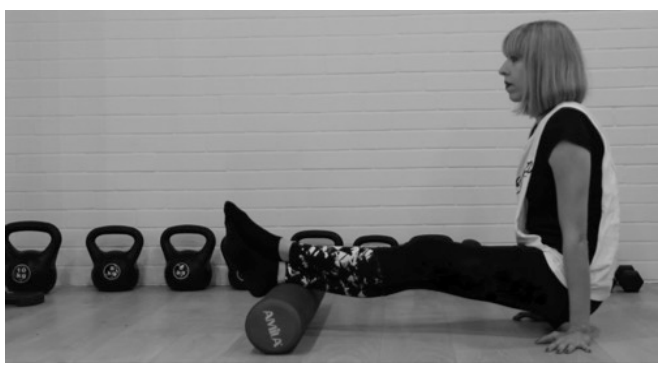

Figure 2. Initial Position of Foam Rolling the ankle plantar flexors 


\subsection{Statistical Analysis}

The analysis of the data was performed with SPSS and statistical significance was set at $>0.05$. Since the control group didn't receive training, the least flexible limb was classified as "treated" and the more flexible as "untreated" for reasons of comparisons.

For the detection of differences between the flexibility of the ipsilateral limb between the experimental and the control group and between the flexibility of the contralateral limb between the experimental and the control group, two way repeated-measures anovas (2X6) were used. The independent variable was the "group" (experimental or control), the dependent variable was "flexibility" and the repeated factors (within-subjects factors) were "time" (i.e. before the treatment and immediately after and $5,10,15,20 \mathrm{~min}$ after the treatment). This analysis was made separately, once comparing the ipsilateral and once the contralateral limbs of the two groups.

For the comparison of the group that performed FR with the dominant leg with the group that performed FR with the non-dominant leg, a repeated measures ANOVA (2x2x6) was used. The independent variable was "limb dominance" (dominant or not dominant limb) and the dependent variable was "flexibility". The repeated factors (within-subjects factors) were "time" (same time points as before) and the "leg" (i.e. ipsilateral/treated leg and contralateral/not treated leg).

When an interaction was found, Sidak pairwise comparisons were performed.

\section{Results}

When comparing the experimental $(\mathrm{n}=19)$ with the control group $(n=19)$ in the flexibility of the treated leg, there was a statistically significant interaction between time* (and) group $\left(\mathrm{F}_{5,180}=8.289, \mathrm{p}=0.000<0.05\right)$. By analyzing the interaction of time for every level of the factor "group", there was documented a statistically significant effect of the factor "time" within the levels of the factor "group" (experimental and control). Specifically, in the experimental group there was a statistically significant effect of the factor "time" in the flexibility of the treated leg $\left(\mathrm{F}_{5,32}=12.907, \mathrm{p}=0.000<0.05\right)$ and as it was revealed by Sidak pairwise comparisons there were statistically significant increases of the flexibility in all time points compared with baseline while in the control group there wasn't a statistically significant increase for any time point compared to baseline $\left(\mathrm{F}_{5.32}=0.732\right.$, $\mathrm{p}=0.605>0.05$ ).

When comparing the experimental with the control group in the flexibility of the untreated leg, it was documented that there was a statistically significant interaction between time* (and) group $\left(\mathrm{F}_{5,180}=3.531\right.$, $\mathrm{p}=0.005<0.05$ ). By analyzing the interaction of time for every level of the factor "group" there was documented a statistically significant effect of the factor "time" within the levels of the factor "group". Specifically, in the experimental group the flexibility of the untreated leg increased significantly immediately after, and 10, 15, and $20 \mathrm{~min}$ after the application and also there was a statistically significant increase between 5 and $20 \mathrm{~min}$ $\left(\mathrm{F}_{5.32}=8.362, \mathrm{p}=0.000<0.05\right)$ while in the control group there wasn't a statistically significant increase for any time point compared to baseline $\left(\mathrm{F}_{5.32}=0.242\right.$, $\mathrm{p}=0.941>0.05)$ (Table 1$)$.

Proceeding with the analysis in the two subgroups that applied FR, there wasn't an interaction between the three defined factors $\left(\mathrm{F}_{5,85}=1.03, \mathrm{p}>.05\right)$. When it was investigated if there was a main effect of the factor "limb dominance" (dominant or non-dominant limb) (between subjects effects), it was shown that there weren't statistically significant differences in the magnitude of the increase of the flexibility of the contralateral limb when the training was done with the dominant or with the non-dominant limb $\left(\mathrm{F}_{1,17}=1.2, \mathrm{p}>.05\right)$. There was found a main effect of the factor "time" $\left(\mathrm{F}_{5,85}=13.96 \mathrm{p}<.05\right)$ that means that the values of the flexibility of the two limbs had significant differences in the different time points, and main effects of the factor "leg" $\left(F_{1,17}=15.07, p<.05\right)$ that means that the limb that received the treatment had a different process in its performance than the untreated leg. Since, in this phase, what was under investigation were the differences between the limbs according to leg dominance, and although there wasn't found neither an interaction nor a main effect of the factor "limb dominance" (dominant or non-dominant limb), the mean values of each limb separately are presented in Table 2, together with their statistical significance.

Table 1. Mean scores of ankle ROM of both legs of experimental and control group (in $\mathrm{cm}$ )

\begin{tabular}{|c|c|c|c|c|c|c|}
\hline \multicolumn{7}{|c|}{ TREATED LEG } \\
\hline & BASELINE & 0 min & 5 min & 10 min & $15 \min$ & 20 min \\
\hline Experimental Group $(n=19)$ & $11.89 \pm 2.62$ & $\mathbf{1 2 . 8 9} \pm \mathbf{2 . 8 7} *$ & $\mathbf{1 2 . 9 4} \pm \mathbf{3 . 0 6} *$ & $\mathbf{1 3 . 1 1} \pm 2.93 *$ & $\mathbf{1 3 . 3 8} \pm \mathbf{3 . 1 2 *}$ & $\mathbf{1 3 . 4 3} \pm \mathbf{3 . 0 0} *$ \\
\hline Control Group $(\mathrm{n}=19)$ & $12.73 \pm 2.62$ & $12.71 \pm 2.69$ & $12.87 \pm 2.62$ & $12.90 \pm 2.67$ & $12.96 \pm 2.76$ & $12.76 \pm 2.78$ \\
\hline \multicolumn{7}{|c|}{ UNTREATED LEG } \\
\hline & BASELINE & 0 min & 5 min & 10 min & 15 min & 20 min \\
\hline Experimental Group $(\mathrm{n}=19)$ & $13.38 \pm 2.87$ & $\mathbf{1 3 . 9 0} \pm \mathbf{2 . 9 9 *}$ & $13.89 \pm 3.14$ & $\mathbf{1 4 . 1 5} \pm \mathbf{3 . 0 4 *}$ & $\mathbf{1 4 . 3 9} \pm \mathbf{3 . 2 4} *$ & $\mathbf{1 4 . 4 7 \pm 3 . 1 0 *}$ \\
\hline Control Group $(\mathrm{n}=19)$ & $13.64 \pm 2.56$ & $13.67 \pm 2.43$ & $13.80 \pm 2.37$ & $13.83 \pm 2.54$ & $13.79 \pm 2.29$ & $13.83 \pm 2.29$ \\
\hline
\end{tabular}


Table 2. Mean scores of ankle ROM of ipsilateral and contralateral limbs, according to leg dominance (in $\mathrm{cm}$ )

\begin{tabular}{|c|c|c|c|c|c|c|}
\hline \multicolumn{7}{|c|}{ DOMINANT LEG GROUP (n=8) } \\
\hline & BASELINE & $0 \mathrm{~min}$ & $5 \min$ & $10 \mathrm{~min}$ & $15 \mathrm{~min}$ & $20 \mathrm{~min}$ \\
\hline $\begin{array}{c}\text { Ipsilateral } \\
\text { limb }\end{array}$ & $12.66 \pm 3.07$ & $13.96 \pm 3.05^{*}$ & $13.76 \pm 3.30$ & $13.87 \pm 2.89 *$ & $14.20 \pm 3.26^{*}$ & $14.08 \pm 3.16^{*}$ \\
\hline $\begin{array}{c}\text { Contralateral } \\
\text { limb }\end{array}$ & $14.47 \pm 2.41$ & $14.84 \pm 2.64$ & $14.61 \pm 2.49$ & $14.92 \pm 2.48$ & $15.27 \pm 2.99$ & $15.34 \pm 2.88$ \\
\hline \multicolumn{7}{|c|}{ NON-DOMINANT LEG GROUP $(n=11)$} \\
\hline & BASELINE & $0 \min$ & $5 \mathrm{~min}$ & $10 \mathrm{~min}$ & $15 \mathrm{~min}$ & $20 \mathrm{~min}$ \\
\hline $\begin{array}{c}\text { Ipsilateral } \\
\text { limb }\end{array}$ & $11.33 \pm 2.23$ & $12.10 \pm 2.59 *$ & $12.34 \pm 2.88^{*}$ & $12.56 \pm 2.96 *$ & $12.78 \pm 3.01 *$ & $12.95 \pm 2.93 *$ \\
\hline $\begin{array}{c}\text { Contralateral } \\
\text { limb }\end{array}$ & $12.60 \pm 3.02$ & $13.23 \pm 3.16$ & $13.37 \pm 3.57$ & $13.58 \pm 3.40 *$ & $13.74 \pm 3.39 *$ & $13.84 \pm 3.24 *$ \\
\hline
\end{tabular}

\section{Discussion}

As it was shown from the statistical analysis, the protocol was effective in producing statistically significant increases of the ROM of both the treated and the untreated leg of the experimental group but not of the control group, proving that these effects were not due to the repetitive weight bearing lunge test but to the FR protocol. Also, there was a CToF both when the dominant and the non-dominant limb received the training but there weren't statistically significant differences in the magnitude of $\mathrm{CToF}$ between the dominant and the non-dominant leg groups.

The increase of flexibility of the ipsilateral limbs (independently of limb dominance) could be explained through the mechanical properties of fascia (i.e. viscoelasticity, trixotrophy). Specifically, it has been supported that application of pressure (i.e. through rolling) or heat (which is also could be provoked by pressure) can alter the viscoelasticity and trixotrophy of muscle and fascia [21,22]. A change in the viscoelastic and trixotrophic properties means that the tissue changes in stiffness [23] and viscosity [24], it becomes softer, more malleable [25] and less resistant when a stretch is applied, permitting, in this way, the achievement of a greater ROM [24]. These effects in the tissues' plasticity should be seen not only in relation to their mechanistic properties, since they alone would fail to totally explain the immediate effects, but also taking into account the contribution of the nervous system. Precisely, it has been argued that mechanical stimulation (e.g. through pressure) of the fascial mechanoreceptors and more importantly of the so-called Ruffini-Endings and Interstitial Receptors, result in global muscle relaxation [26] and this could also explain the increases in the flexibility both of the ipsilateral and of the contralateral limb of the experimental group. The fact that the contralateral limbs weren't mechanically loaded supports the hypothesis that their adaptations could be explained mainly via the neural system.

The ipsilateral limbs of both of the subgroups (dominant leg group and non-dominant leg group) experienced statistically significant increases in the flexibility in the majority of the time points (Table 2). When comparing them, though, in the flexibility of the contralateral limbs, there weren't found statistically significant differences in the magnitude of the CToF, implying that when referring to CToF after FR the transfer is not AT (at least according to the methodological design of the present study that compared two different groups who trained their least flexible limb). This finding adds new information to the existing literature, providing some initial suggestions and new data about $\mathrm{CToF}$ of the lower limbs in relation to laterality. Taking under consideration that strength transfer and skill transfer are thought to be fundamentally different but also closely connected [1], it could be postulated that CToF should be seen as a different entity, which could share some similarities and also carry some differences from CT of strength or skills (for a review of CT of strength and skills see 1,6).

Firstly, as it is pointed out, when studying limb dominance in relation to $\mathrm{CT}$ and $\mathrm{AT}$, it would not be advisable to compare directly the upper with the lower limbs, because they have differences in their function and also they differ in their motor strategies as it is documented from their relative cortical activities (i.e. the lower limbs operate with more cross-hemispheric interaction and less independently than the upper limbs). Concerning the lateralization of the lower limbs (i.e. footedness) for strength or skills, it is probably less prominent than handedness $[1,8]$, since there is more or less an equal contribution of both legs in the normal daily activities [1], the most proficient limb varies according to the context [8] and depends on the characteristics and the specific requirements of the activities and the tasks $[1,9,10]$.

Taking into account the above-mentioned data and in order to establish an explanation of the findings of the present research, initially, it should be noticed that according to baseline measures, leg dominance was not related with greater or smaller proficiency in the flexibility of the ankle plantar flexors (in 11 gym 
members the most flexible limb was the non-dominant and in 8 the dominant). As it has been suggested, in the lower limbs, the dominant leg is specialized in mobilization or (object) manipulation tasks and involves ROM while the non-dominant leg in stabilization and requires isometric force control [9]. However, in foam rolling the ankle plantar flexors and then controlling their flexibility through a simple in the execution weight bearing lunge test, there wouldn't be probable reasons to expect that the limb that is preferred for mobilizing tasks (dominant) would be more proficient or more receptive in an increase of its flexibility than the limb that is preferred for stabilizing tasks (non-dominant) and vice versa. Since the protocol was equally effective in increasing the flexibility of the ipsilateral dominant or non-dominant limbs and taking into account that the contralateral increases of flexibility were probably due to the effect of pressure at the autonomic nervous system (which influences the muscle tonus regulation of the entire body) $[27,28]$, the fact that there was not a preferential side of CToF seems logical.

Additionally, when referring to $\mathrm{CT}$ of skills (i.e. the transfer of an acquired motor skill from the limb that "learned" it first to the other) it has been supported that it is symmetric in simple tasks and asymmetric in more complex movements and it may be related to hemispheric specialization [8]. Also, as it has been supported for upper limbs, if a task is unfamiliar to both limbs, transfer will probably occur bilaterally [5]. In the present study the exercise protocol (FR) was different from the measurements executed (weight-bearing lunge test), intervention and test were performed in one single session, and both of them were novel for the participants but not complex in their execution. Also, the samples of the present research were female gym members, a population that, contrary to athletes of certain sports, use both legs simultaneously in activities of their daily life, such as locomotion [29] and also, being exercisers, in the gyms they practice more or less equally both legs in the distinct parameters of the general physical condition (e.g. strength, flexibility etc.). For these reasons, and considering also that the slow and melting and steady deep pressure provoked by the FR could increase parasympathetic activity, lowering the muscle tonus of the entire body $[27,28]$ and subsequently of the contralateral muscles, and considering that such neural adaptations is not be said to be side-specific, we conclude that when referring to the enhancement of contralateral flexibility through FR the transfer will probably occur symmetrically.

\section{Conclusions}

The FR protocol that was used in the present research was effective in increasing the ipsilateral and contralateral ankle ROM in female gym members independently of limb dominance, revealing that when referring to $\mathrm{CToF}$ after FR, AT is not present. These preliminary findings should be further expanded through the conduction of larger-scale researches with larger samples, for more sessions, and if possible with persons who suffer a unilateral injury. If it is established that injured persons could gain benefit from CToF independetely of limb dominance, that would be a major contribution to the field of rehabilitation.

\section{Acknowledgements}

The research work was supported by the Hellenic Foundation for Research and Innovation (HFRI) under the HFRI PhD Fellowship grant (Fellowship Number: 474). The authors would also like to thank all the gym members for their participation in the research and the owners of the gyms for the kind provision of adequate spaces in the gyms in order to hold the intervention. The photo-shootings of the figures were realized at the gyms "Point of You Fitness Hall" and "Cocoon Personal Fitness".

\section{REFERENCES}

[1] J. P. Farthing. Cross-education of strength depends on limb dominance: Implications for theory and application, Exercise and Sport Sciences Reviews, Vol.37, No.4, 179187, 2009.

[2] S. Kelly, C. Beardsley. Specific and Cross-Over Effects of Foam Rolling on Ankle Dorsiflexion Range of Motion, The International Journal of Sports Physical Therapy, Vol.11, No.4, 544-551, 2016.

[3] D. J. Kidgell, A. K. Frazer, A. J. Pearce. The Effect of Task Complexity Influencing Bilateral Transfer, International Journal of Exercise Science, Vol.10, No.8, 1174-1183, 2017.

[4] S. E. Parlow, M. Kinsbourne. Asymmetrical transfer of training between hands: Implications for interhemispheric communication in normal brain. Brain and Cognition, Vol.11, No.1, 98-113, 1989.

[5] J. P. Farthing, P. D. Chilibeck, G. Binsted. Cross-education of arm muscular strength is unidirectional in right-handed individuals, Medicine and Science in Sports and Exercise, Vol.37, No.9, 1594-1600, 2005.

[6] A. M. Hendy, M. Spittle, D. J. Kidgell. Cross education and immobilisation: Mechanisms and implications for injury rehabilitation, Journal of Science and Medicine in Sport, Vol.15, No.2, 94-101, 2012.

[7] T. A. Coombs, A. K. Frazer, D. M. Horvath, A. J. Pearce, G. Howatson, D. J. Kidgell. Cross-education of wrist extensor strength is not influenced by non-dominant training in right-handers, European Journal of Applied Physiology, Vol.116, No.9, 1757-1769, 2016. 
[8] T. Morris, N. A. Newby, M. Wininger, W. Craelius Inter-limb transfer of learned ankle movements. Experimental Brain Research, Vol.192, No.1, 33-42, 2009.

[9] S. C. Yen, L. C. Olsavsky, C. M. Cloonan, A. R. Llanos, K J. Dwyer, M. Nabian, A. B. Farjadian. An examination of lower limb asymmetry in ankle isometric force control, Human Movement Science, vol.57, 40-49, 2018.

[10] H. J. A. van Hedel, M. Biedermann, T. Erni, V. Dietz. Obstacle avoidance during human walking: Transfer of motor skill from one leg to the other, Journal of Physiology, Vol.543, No.2, 709-717, 2002.

[11] C. Krishnan, R. Ranganathan, M. Tetarbe. Interlimb transfer of motor skill learning during walking: No evidence for asymmetric transfer, Gait and Posture, Vol. 56, 24-30, 2017.

[12] J. P. Farthing, J. R. Krentz, C. R. A. Magnus. Strength training the free limb attenuates strength loss during unilateral immobilization, Journal of Applied Physiology, Vol.106, No.3, 730-736, 2009

[13] C. Wend. The Effects of Limb Dominance on CrossEducation in a Four Week Resistance Training Program. Master Theses. 2885. Eastern Illinois University, 2017. Online available from http://thekeep.eiu.edu/theses/2885.

[14] A. Chaouachi, J. Padulo, S. Kasmi, A. Ben Othmen, M. Chatra, D. G. Behm. Unilateral static and dynamic hamstrings stretching increases contralateral hip flexion range of motion, Clinical Physiology and Functional Imaging, Vol. 37, No.1, 23-29, 2017.

[15] M. T. García-Gutiérrez, P. Guillén-Rogel, D. J. Cochrane, P. J. Marín. Cross transfer acute effects of foam rolling with vibration on ankle dorsiflexion range of motion, Journal of Musculoskeletal and Neuronal Interactions, Vol.18, No.2, 262-267, 2018.

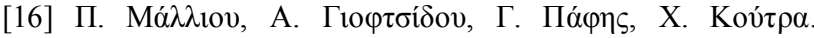

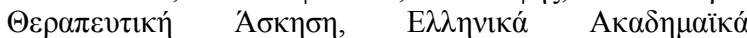

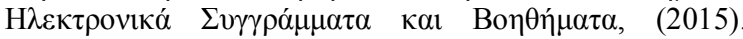
Online available at: http://hdl.handle.net/11419/372

[17] N. van Melick, B. M. Meddeler, T. J. Hoogeboom, M. W. G. Nijhuis-van der Sanden, R. E. H. van Cingel. How to determine leg dominance: The agreement between self-reported and observed performance in healthy adults. PLoS ONE, Vol.12, No.12, 1-9, 2017.

[18] K. Bennell, R. Talbot, H. Wajswelner, W. Techovanich, D. Kelly. Intra-rater and inter-rater reliability of a weight-bearing lunge measure of ankle dorsiflexion, Australian Journal of Physiotherapy, Vol.44, No.3, 175$180,1998$.
[19] I. Halperin, S. J. Aboodarda, D. C. Button, L. L. Andersen, D. G. Behm. Roller massager improves range of motion of plantar flexor muscles without subsequent decreases in force parameters, The International Journal of Sports Physical Therapy, Vol.9, No.1, 92-102, 2014.

[20] J. Škarabot, C. Beardsley, I. Štirn. Comparing the effects of self-myofascial release range-of-motion in adolescent athletes, The International Journal of Sports Physical Therapy, Vol.10, No.2, 203-212, 2015.

[21] D. J. Bradbury-Squires, J. C. Noftall, K. M. Sullivan, D. G. Behm, K. E. Power, D. C. Button. Roller-massager application to the quadriceps and knee-joint range of motion and neuromuscular efficiency during a lunge, Journal of Athletic Training, Vol.50, No.2, 133-140, 2015.

[22] S. W. Cheatham, M. J. Kolber, M. Cain. Comparison of Video-Guided, Live Instructed, and Self-Guided Foam Roller Interventions on Knee Joint Range of Motion and Pressure Pain Threshold: A Randomized Controlled Trial, The International Journal of Sports Physical Therapy, Vol.12, No.2, 242-249, 2017.

[23] H. M. Langevin. Connective tissue: A body-wide signaling network? Medical Hypotheses, Vol.66, 1074-1077, 2006.

[24] A. R. Mohr, B. C. Long, C. L. Goad. Foam Rolling and Static Stretching on Passive Hip Flexion Range of Motion. Effect of Foam Rolling and Static Stretching on Passive Hip-Flexion Range of Motion, Journal of Sport Rehabilitation, Vol.23, 296-299, 2014.

[25] S. N. Madoni, P. B. Costa, J. W. Coburn, A. J. Galpin. Effects of Foam Rolling on Range of Motion, Peak Torque, Muscle Activation, and the Hamstrings-to-Quadriceps Strength Ratios, The Journal of Strength \& Conditioning Research, Vol.32, No.7, 1821-1830, 2018.

[26] R. Schleip. Fascia as a Sensory Organ. In E. Dalton (Ed.), Dynamic Body - Exploring Form, Expanding Function, Oklahoma: Freedom from Pain Institute, 2011.

[27] R. Schleip, R. Fascial plasticity - a new neurobiological explanation Part 1, Journal of Bodywork \& Movement Therapies, Vol.7, No.1, 11-19, 2003.

[28] R. Schleip. Fascial plasticity - a new neurobiological explanation Part 2. Journal of Bodywork \& Movement Therapies, Vol.7, No.2, 104-116, 2003.

[29] Valdez, D. (2003). Bilateral Asymmetries in Flexibility, Stability, Power, Strength, and Muscle Endurance Associated with Preferred and Nonpreferred Leg. Master Theses. University of Florida. Available online from: https://pdfs.semanticscholar.org/f057/31e80dd3626651e20 ac0f9845287ddeee4ee.pdf 